\title{
Pyrethroid Insecticides-Time for a Closer Look
}

Steven D. Stellman, PhD, MPH; Jeanne Mager Stellman, PhD

Pyrethroid pesticides are a large family of synthetic analogues of naturally occurring pyrethrins widely used for insect control in agricultural and numerous consumer products. Collectively, they are the second most-used insecticides in the world, totaling thousands of kilograms and billions of dollars in US sales.

In this issue of JAMA Internal Medicine, a group of researchers from the University of Iowa College of Public Health compared total and cardiovascular deaths among participants in 2 biennial cycles of the highly regarded National Health and Nu-

$+$

Related article trition Examination Survey (NHANES), which includes a nationally representative sample of US adults. The researchers followed the participants for up to 16.8 years after urinary measurement of 3-phenoxybenzoic acid (3-PBA), a metabolite of pyrethroid exposure. ${ }^{1}$ They reported significant hazard ratios of 1.56 for death from any cause and 3.00 for death from cardiovascular disease for individuals in the highest compared with the lowest biomarker exposure tertile.

Given the widespread use of pyrethroids and numerous exposure assessments in the general population, including in children ${ }^{2,3}$ and occupationally exposed individuals, ${ }^{4}$ the results of this study warrant immediate further investigation, especially because pyrethroids have long been considered of minimal hazard to humans and play a vital role in public health control of vector-borne illnesses.

Further, unlike organochlorine pesticides, such as DDT and dieldrin, which persist in adipose tissue for decades, the biomarker 3-PBA has a very short half-life, as low as 5.7 hours. ${ }^{5}$ The prevalence of detectable levels of a rapidly eliminated pyrethroid metabolite in a large, geographically diverse population is suggestive of chronic exposure, which also makes it important to identify specific environmental sources.
However, caution is needed in interpreting this study.

First, data were available only on adults aged $20-59$ years at baseline, so that the average age at the end of follow-up was approximately 57 years, which is young for assessing cardiovascular mortality effects. Other than cigarette smoking, few, if any, chemical exposures are known to trigger a 3-fold increase in the risk of death from heart disease, especially in persons younger than 60 years, suggesting that other, as yet unknown, factors may be associated with these increased risks.

Second, while cardiovascular effects associated with pyrethroid exposure have been observed experimentally in rodents and other animals, ${ }^{4}$ to date there is little epidemiological evidence to suggest such effects in humans. A single case-control study of coronary heart disease cited by the authors reports an odds ratio of 3.6 for the upper compared with the lower tertile of 3-PBA; in that study, the average age of participants with coronary heart disease was 62.5 years, or only about 5 years older than participants in the NHANES, while the biomarker levels were considerably greater than those in the NHANES population. ${ }^{6}$

Further detailed studies based on validated exposure assessment methods, including both questionnaires and biomarkers, are needed to assess these toxicological parameters.

Pyrethroid pesticides are ubiquitous, and exposure is unavoidable; in New York City and elsewhere, aerial spraying for mosquito control to prevent West Nile virus and other vector-borne illnesses is largely based on pyrethroids. ${ }^{7}$ This study challenges the assumption that such exposures are safe. The unusually large hazard ratios observed deserve immediate further exploration, which would contribute to more evidence-based options in weighing risks and benefits of essential insect control programs.

\section{ARTICLE INFORMATION}

Author Affiliations: Department of Epidemiology, Mailman School of Public Health, Columbia University, New York, New York (S. D. Stellman); Department of Health Policy and Management, Mailman School of Public Health, Columbia University, New York, New York (J. M. Stellman).

Corresponding Author: Steven D. Stellman, PhD, $\mathrm{MPH}$, Department of Epidemiology, Mailman School of Public Health, Columbia University, New York, NY (sds91@columbia.edu).

Published Online: December 30, 2019. doi:10.1001/jamainternmed.2019.6093

\section{Conflict of Interest Disclosures: None reported.}

\section{REFERENCES}

1. Bao W, Liu B, Simonsen DW, Lehmler H-J. Association between exposure to pyrethroid insecticides and risk of all-cause and cause-specific mortality in the general US adult population [published online December 30, 2019]. JAMA Intern Med. doi:10.1001/jamainternmed.2019.6019

2. Barr DB, Olsson AO, Wong LY, et al. Urinary concentrations of metabolites of pyrethroid insecticides in the general US population: National Health and Nutrition Examination Survey 1999-2002. Environ Health Perspect. 2010;118(6): 742-748. doi:10.1289/ehp.0901275

3. Naeher LP, Tulve NS, Egeghy PP, et al. Organophosphorus and pyrethroid insecticide urinary metabolite concentrations in young children living in a southeastern United States city. Sci Total Environ. 2010;408(5):1145-1153. doi:10.1016/j. scitotenv.2009.10.022

4. US Department of Health and Human Services, Public Health Service, Agency for Toxic Substances and Disease Registry. Toxicological Profile for
Pyrethrins and Pyrethroids. Atlanta, GA: Agency for Toxic Substances and Disease Registry; 2003.

5. Ratelle M, Cote J, Bouchard M. Toxicokinetics of permethrin biomarkers of exposure in orally exposed volunteers. Toxicol Lett. 2015;232(2):369375. doi:10.1016/j.toxlet.2014.12.003

6. Han J, Zhou L, Luo M, et al. Nonoccupational exposure to pyrethroids and risk of coronary heart disease in the Chinese population. Environ Sci Technol. 2017;51(1):664-670. doi:10.1021/acs.est.6b05639

7. New York City Department of Health and Mental Hygiene. Mosquito control events. NYC.gov website. https://www1.nyc.gov/site/doh/health/ health-topics/west-nile-virus-spray.page. Published 2019. Accessed October 12, 2019. 